\title{
Predicting the shelf life of milk powder
}

\author{
V. Chopovda ${ }^{1} \quad$ R. J. Clarke ${ }^{2} \quad$ A. C. Fowler ${ }^{3}$ \\ L. A. Fullard ${ }^{4}$ J. Goodman ${ }^{5}$ L. M. Thomasen ${ }^{6}$ \\ S. W. Taylor ${ }^{7}$
}

(Received 23 October 2017; revised 23 October 2018)

\begin{abstract}
The aim of this paper is to gain an understanding of the factors that influence the degradation of bags of milk powder when they are shipped around the world. We discuss two approaches, mathematical modelling and experimental design. We model the spatial and temporal variation of humidity, oxygen and temperature and its effect on the shelf life of the bags of milk powder. We show that the diffusion of these quantities is so rapid that they can be assumed to be uniform throughout the bags. This justifies the simplification of the model to the form of a system of ordinary differential equations involving time. The experimental design discussed in this paper involves a statistical approach to measuring the different factors that influence the degradation of milk powder over time. The mathematical modelling will allow industries to calculate
\end{abstract}

DOI:10.21914/anziamj.v58i0.12554, (C) Austral. Mathematical Soc. 2018. Published 2018-12-04, as part of the Proceedings of the 2016 Mathematics and Statistics in Industry NZ Study Group. ISSN 1445-8810. (Print two pages per sheet of paper.) Copies of this article must not be made otherwise available on the internet; instead link directly to the DOI for this article. 
how quickly specific environmental conditions lead to milk degradation while it is in transit. The aim of the experimental design is to provide a means to measure the effects of environmental conditions on milk powder. It is impossible to collect such data on actual milk powder shipments.

Subject class: 93A30; 62P30

Keywords: milk powder, shelf life, glass transition, experimental design

\section{Contents}

1 Introduction

2 The effect of diffusion

2.1 Thermal transport ................ . M385

2.2 Moisture transport ................. . M386

2.3 Oxygen transport ................ M391

3 Permeation model $\quad$ M391

3.1 Assumptions ..................... M392

3.2 Model equations ................... M393

3.3 Results...................... . . M395

3.3.1 Constant temperature and humidity . . . . . . . M395

3.3.2 Example measured temperature . . . . . . . . . M398

4 Using compaction to reduce milk powder spoilage $\quad$ M400 4.1 Compression .................... . M402 4.2 Vibro-compaction ................. M404

5 Statistical approaches $\quad$ M406 5.1 Customer feedback data . . . . . . . . . . . . . . M406 5.1.1 Age ................. M406 
5.1 .2 Country . . . . . . . . . . . . . M408

5.2 Experimental design . . . . . . . . . . . . . . . . M408

5.2 .1 Factors . . . . . . . . . . . . . . . M409

5.2 .2 Pilot study . . . . . . . . . . . . . . . . . . . M409

5.2 .3 Logistics . . . . . . . . . . . . . . . . M410

5.2 .4 Time ............................ M411

\section{Introduction}

Milk powder, in all of its varieties, is a complex bulk material. Fonterra, New Zealand, produces on the order of 1.5 MT of powder annually, which includes all whole, skim, and buttermilk powders. These products are shipped and stored around the world for consumer use, as well as being used as an ingredient in the food industry. Maintaining the quality of the powder during transport and storage is of utmost importance, since even when the powder is still safe to eat, various other sensory factors, such as colour, taste, or smell, may result in rejection of the product by the consumer. The main problems which arise over time, and ultimately determine powder quality, are lipid oxidation, lactose crystallisation and Maillard reactions (non-enzymatic browning) [24, 22]. All are dependent on the temperature and moisture content of the powder.

Lipid oxidation, lactose crystallisation, and Maillard reactions are governed by a large number of chemical reactions [23], the formulation and solution of which was not possible during the limited time available during Minz. However, we discuss these in a little more detail. During powder processing, steps are taken to minimise the effect of these three processes. During bagging, air is removed from the powder by inserting a lance which pumps a mixture 
of carbon dioxide and nitrogen gas into the bag. These gases are very stable and do not react with the powder. Additionally, the reduction in oxygen from the air reduces the amount of lipid oxidation. Such a procedure is known to greatly extend the shelf life of milk powders [17, 22]. However, there is still some residual oxygen left inside the bag of powder, both in the bag head-space, and trapped between the particles (which will migrate due to diffusion and agitation). Additionally, for the type of bags used to store milk powder, some oxygen will permeate through the bag material. Preliminary calculations approximate this rate as around $5 \%$ of the initial oxygen level per year. Furthermore, the rate of lipid oxidation is highly dependent on the water activity, $a_{w}$, which is defined as the ratio of the vapour pressure of water in the food product to the vapour pressure of pure water (at the current temperature). In food science the use of water activity is preferred over the term relative humidity (which is reserved for the vapour pressure ratio in air). The rate of lipid oxidation reaches a minimum at roughly around $0.2<\mathrm{a}_{w}<0.4$ [15]. Hence, to delay oxidation, Fonterra aims to dry the powder to such a range. Reducing the water content is a costly process, so it is desirable to reduce the level no more than necessary. This level depends on the target market, with lower values of $\mathrm{a}_{w}$ preferred in hot humid regions. However, reducing the initial $a_{w}$ below $\approx 0.2$ is counter productive, as the rate of lipid oxidation rapidly increases for $a_{w}<0.2$ [15].

Maillard reactions are induced by heating during the powder forming process, or during long-term storage at elevated temperatures [24]. Such reactions cause browning of the powder which is aesthetically displeasing. The method and intensity of heating helps reduce the effect of Maillard reactions [21].

Lactose crystallisation occurs once the temperature of the powder increases above the glass transition temperature, $T_{g}$, until it reaches the melting point of the powder. The glass transition temperature decreases as the water activity, $a_{w}$, increases. Figure 1 shows that the dependence is approximately linear. Once the glass transition temperature has been exceeded then lactose crystallisation occurs, which releases chemically bound water [24], further increasing the rate of Maillard reactions, and further accelerating the chemical 
reactions which degrade the quality of the powder. In practice, the amount of water in the powder is reduced so that the system is ensured to be far from the glass transition temperature. However, over time moisture may permeate the bag packaging, increasing the water activity, and driving the system closer to the glass transition curve.

Much of the customer feedback data provided by Fonterra was from hot and humid countries. Humidity allows greater levels of permeation of moisture into the bag, accelerating all of the described reactions. Furthermore, an increase in the ambient temperature accelerates the rate of chemical reactions, and pushes the powder closer to the glass transition temperature, increasing the danger that lactose crystallises. Furthermore, the discussed reactions are exothermic [24], hence, once a reaction initiates, the rates of reactions may accelerate, leading to further reactions and degrading of powder quality. This reaction generated internal heating may be a larger issue when the bag is a part of a pallet, since the extra heat may not be efficiently radiated to the atmosphere.

It is evident from the above discussion that prediction of the shelf life of milk powders is a complicated and highly non-linear problem. Given sufficient experimental data, time, and resources, the main reactions could be formulated into a kinetic reaction model with temperature dependent reaction rate (Arrhenius-type dependence). However, since the MINZ meeting was less than a week, to make some headway on this problem we considered a series of smaller sub-problems.

Time scales for heat and moisture transport through the powder are discussed in Section 2. Calculation of the diffusion of moisture through the bag membrane and powder pellets is considered as a way to approximate when the powder reaches the glass transition temperature, and hence, approximate the shelf life of the product. An analytical solution is provided, in contrast to the numerical solutions of Section 3.

In Section 3 we model the mass transfer of water vapour through the packaging membrane in an attempt to predict how storage conditions affect shelf life of 
the powder. Crystallisation of lactose occurs once the temperature and water activity of the powder sample exceeds the critical glass temperature (which is dependent on $a_{w}$ ). Before any significant reactions occur in the powder, the amount of moisture in the bag increases mainly by permeation over the bag membrane. This allows us to formulate a mass transfer model which predicts the time when the powder surpasses the glass transition temperature, and hence, begins lactose crystallisation. This time measures how long it takes for some of the negative reactions to initiate, although the rates of oxidation and Maillard reactions should be included in further work to obtain a more accurate measure of shelf life. The model allows a calculation of shelf life based on the ambient conditions. Mass transfer models across the bag membrane, in the powder itself, and due to uneven heating on opposite sides of the powder sample have been discussed previously [1, 2, 6, 3, 5, 13].

Oxygen is a key reactant in lipid oxidation. Labuza and Dugan [15] discuss oxygen's effect on the rate of lipid oxidation. The method currently used to slow lipid oxidation is to limit the amount of oxygen present by displacing it with the inert gas nitrogen. We suggest another method in Section 4, where we review literature on compression and vibro-compaction of powder beds in an effort to reduce the pore space, and hence, the initial packed oxygen content of the bag of milk powder. Such a procedure has the potential to lengthen the shelf life of the product.

Finally, Section 5 applies statistical analysis to Fonterra customer feedback data accounting for various factors including country of shipment, powder type, date of manufacture and complaint and various other factors. We also design a fractional factorial split plot set of experiments to allow Fonterra to further understand the effect of various factors on the shelf life of milk powders. 


\section{The effect of diffusion}

In this section, we develop a model to approximate thermal and diffusive time scales in the powder system. Our estimates of diffusivities confirm that diffusion is rapid enough to allow us to simplify models, removing spatial effects, similar to the model discussed in Section 3. The simple model that we obtain allows us to develop explicit formulae for shelf life of milk powder.

We consider the transport of heat and moisture through sealed bags of milk powder. A $25 \mathrm{~kg}$ bag has dimensions of $830 \times 430 \times 130 \mathrm{~mm}$, and is sealed by a membrane whose construction varies in order to limit the transport of moisture. Degradation reactions are generally heat and moisture dependent, and the primary issue in controlling them appears to be the effort to maintain the powders in a dry and cool environment. When the moisture level becomes too high, a glass transition occurs and this leads to various problems such as caking and browning, as discussed by Roos [20].

Another issue is flavour deterioration, due to oxidation of fats. The reaction rate is thermally activated (a doubling every $10^{\circ} \mathrm{C}$ ) and also exothermic. To prevent such reactions, the powder is gas-packed with inert gases $\left(\mathrm{N}_{2}\right.$ and $\mathrm{CO}_{2}$ ). Degradation will thus occur due to diffusion of oxygen through the bag. This problem is similar to that for moisture transport.

\subsection{Thermal transport}

The thermal time scale is $t_{T}=d^{2} / \kappa$, where $d$ is bag dimension and $k$ is thermal diffusivity. For $\mathrm{d} \sim 0.13 \mathrm{~m}$ and $\mathrm{k} \sim 10^{-5} \mathrm{~m}^{2} \mathrm{~s}^{-1}$ (the value for air is $\left.1.9 \cdot 10^{-5} \mathrm{~m}^{2} \mathrm{~s}^{-1}\right), t_{\mathrm{T}} \sim 0.5$ hour. ${ }^{1}$ We therefore assume the temperature in the sample is uniform through the bag. More generally, the temperature is influenced by the day/night cycle, and an average value is relevant.

${ }^{1}$ In reality $t_{T}$ is smaller than this, as the e-folding time for diffusive relaxation is $d^{2} /\left(\pi^{2} \kappa\right)$, a factor of ten smaller. 


\subsection{Moisture transport}

We consider the transport of water vapour through the porous mass of pellets, which we take also to be porous. The simplest model for moisture transport in the bag is a diffusion equation with a sink term, representing diffusive loss to the porous pellets, thus

$$
p_{\mathrm{t}}=\mathrm{D} \nabla^{2} \mathrm{p}-\mathrm{sq}
$$

in the bag $V$, where $s$ is specific surface area, $p$ is water vapour partial pressure, $\mathrm{D}$ is a suitable diffusion coefficient, and $\mathrm{q}$ is the vapour flux to the pellets. The specific surface area is of the form

$$
s=\frac{k}{d_{p}}
$$

where $k$ is constant and $d_{p}$ is pellet diameter. For packed spheres, for example, $k=\pi$. The external boundary condition at the bag surface is

$$
D \frac{\partial p}{\partial n}=h_{b}\left(p_{a v}-p\right)
$$

where $h_{b}$ is a mass transfer coefficient and $p_{a v}$ is the external vapour pressure. The diffusion of water vapour into the pellets is governed by

$$
\frac{\partial p_{p}}{\partial t}=D_{p} \nabla^{2} p_{p} \quad \text { in } \quad V_{p}
$$

where $p_{p}$ is pellet vapour pressure, and $D_{p}$ is a suitable diffusion coefficient in the pellets. The boundary conditions at the surface are

$$
p_{p}=p, \quad D_{p} \frac{\partial p_{p}}{\partial n}=q \quad \text { on } \quad \partial V_{p}
$$

the extra condition providing the definition of $\mathbf{q}$ for PDE (1). 
The time scales for diffusion in the bag and for diffusion in the pellet are respectively

$$
t_{b}=\frac{d^{2}}{D}, \quad t_{p}=\frac{d_{p}^{2}}{D_{p}} .
$$

To estimate these, we take $\mathrm{D} \sim 10^{-5} \mathrm{~m}^{2} \mathrm{~s}^{-1}$ (the diffusivity of water vapour in air is $2.8 \cdot 10^{-5} \mathrm{~m}^{2} \mathrm{~s}^{-1}$ ) and $\mathrm{d} \sim 0.13 \mathrm{~m}$, thus $\mathrm{t}_{\mathrm{b}} \sim 0.5$ hour as for the thermal time scale. The drying time for small powder samples in an oven is five hours, and this provides a (generous) upper limit for $t_{p}$. If $D_{p}=D$, then the time scale would be much smaller, but it seems reasonable to suppose that the effective diffusivity $\mathrm{D}_{\mathrm{p}} \ll \mathrm{D}$ on the basis that the tortuosity of the grains is larger than that of the paths through the mass of pellets, and the pellet porosity is much smaller.

To be specific, let us therefore suppose that $t_{p} \ll t_{b}$. In this case the pellet vapour pressure goes to an approximate equilibrium in which, therefore, $p_{p} \approx p$ is uniform through the pellet. In this case the pellet flux $q$ is approximately zero, and to calculate it we apply a correction to the diffusion PDE (4) of the form

$$
\mathrm{D}_{\mathrm{p}} \nabla^{2} \mathrm{p}_{\mathrm{p}} \approx \mathrm{p}_{\mathrm{t}} .
$$

To be specific let us suppose that the pellets are spherical; then

$$
p_{p} \approx p+\frac{\left(r^{2}-\frac{1}{4} d_{p}^{2}\right) p_{t}}{6 D_{p}}
$$

where $r$ is radius, and therefore

$$
\mathrm{q} \approx \frac{1}{6} \mathrm{~d}_{\mathrm{p}} \mathrm{p}_{\mathrm{t}}
$$

Thus the sink provides an effective reduced diffusivity for the diffusive process in the bag, so that PDE (1) becomes

$$
\left[1+\frac{1}{6} k\right] p_{t}=D \nabla^{2} p,
$$

where we use (2) for the specific surface area. This is known as retarded diffusion, and is most usually encountered when a solute diffuses through 
a porous medium, but also rapidly adsorbs to, and desorbs from, the solid particles of the medium. The restriction to spherical particles is inessential.

Next we estimate the mass transfer coefficient $\boldsymbol{h}_{\mathrm{b}}$. To do this we use that in a normal environment of $70 \%$ humidity in New Zealand, a bag of powder increases its weight fraction by $0.2 \%$ in a year. For a $25 \mathrm{~kg}$ bag, this represents an influx of $0.05 \mathrm{~kg} /$ year, and with a vapour density of $0.8 \mathrm{~kg} \mathrm{~m}^{-3}$, this yields a volume flux of $0.063 \mathrm{~m}^{3}$ /year. The bag surface area is $1 \mathrm{~m}^{2}$, and so the velocity of the moisture through the bag is

$$
h_{\mathrm{b}} \sim 0.063 \mathrm{~m} / \text { year } \sim 2 \cdot 10^{-9} \mathrm{~m} \mathrm{~s}^{-1} .
$$

When scaled, the dimensionless parameter representing the transfer coefficient is thus $h_{b} d / D \sim 2.6 \cdot 10^{-5}$ and is small. Hao, Lu, and Wang [9] show that leakage through the bag is thermally activated, with a typical activation energy of the order of $26-28 \mathrm{~kJ} \mathrm{~mol}^{-1}$. A $40^{\circ} \mathrm{C}$ range would change $h_{\mathrm{b}}$ by a factor of $\sim 4$.

It follows that on a time scale of hours, just as for the pellets, the big vapour pressure is approximately a function of only time, which we denote by $p_{b v}(t)$. The correction to this approximation, which gives the spatial dependence is, from (10),

$$
\mathrm{D} \nabla^{2} \mathrm{p} \approx\left(1+\frac{1}{6} \mathrm{k}\right) \frac{\mathrm{d} \mathrm{p}_{\mathrm{bv}}}{\mathrm{dt}},
$$

together with the boundary condition (3). For a bag of width $\mathrm{d}$, we solve this in one dimension, and then conservation of water vapour, together with (3), leads to the differential equation for the bag vapour pressure,

$$
\left(1+\frac{1}{6} k\right) d \frac{d p_{b v}}{d t}=2 h_{b}\left(p_{a v}-p_{b v}\right) .
$$

Since $h_{b}$ is so small, a time-varying $p_{a v}$ is accommodated by taking a local time average.

The simplest situation is where the bags are stored at a constant temperature $T$ and relative humidity $h_{0}=p_{\mathrm{av}} / p_{\mathrm{sv}}$, measured as a fraction between 0 and 1 ; 
here $p_{\mathrm{sv}}$ is the saturation vapour pressure. Since the bags are initially almost dry, the solution of (13) is just

$$
p_{\mathrm{bv}}=p_{\mathrm{av}}\left[1-\exp \left(-\frac{2 h_{\mathrm{b}} \mathrm{t}}{\left(1+\frac{1}{6} \mathrm{k}\right) \mathrm{d}}\right)\right],
$$

and degradation eventually sets in if $p_{a v}>p_{c}$, where $p_{c}$ is the critical water vapour pressure where the glass transition occurs.

Figure 1 plots the variation of the glass transition temperature as a function of water activity, that is, relative humidity. Such curves are given, for example, by Jouppila and Roos [11] and Thomas et al. [23]. The fitting curve gives

$$
a_{w}=\frac{p_{c}}{p_{s v}}=a_{0}\left(1-\lambda T_{g}\right)
$$

where for the fit shown in Figure 1,

$$
\mathrm{a}_{0}=\frac{1}{2}, \quad \lambda=\frac{1}{90} .
$$

This is similar to the approximation that we use later in (23), the difference being that here we have chosen a convenient rational approximation to the least squares fitting constants.

Motivated by (15), we define the critical relative humidity as

$$
h_{c}=a_{0}(1-\lambda T)
$$

then the glass transition is never reached if $h_{0}<h_{c}$. Figure 1 shows that this is unlikely ever to occur in practice. We assume, therefore, that $h_{0}>h_{c}$, and estimate the shelf life $t_{\mathrm{SL}}$ by finding the time from (14) when $a_{w}$ reaches $h_{c}$; this is

$$
t_{\mathrm{SL}}=\frac{\left(1+\frac{1}{6} k\right) d}{2 h_{b}} \log \left[\frac{h_{0}}{h_{0}-h_{c}}\right]=\frac{\left(1+\frac{1}{6} k\right) d}{2 h_{b}} \log \left[\frac{h_{0}}{h_{0}-a_{0}(1-\lambda T)}\right] .
$$


Figure 1: The glass transition curve. Data from Jouppila and Roos [11], comprising data sets of skim, low fat and whole milk powders as well as pure lactose. The fitting curve is shown in blue.

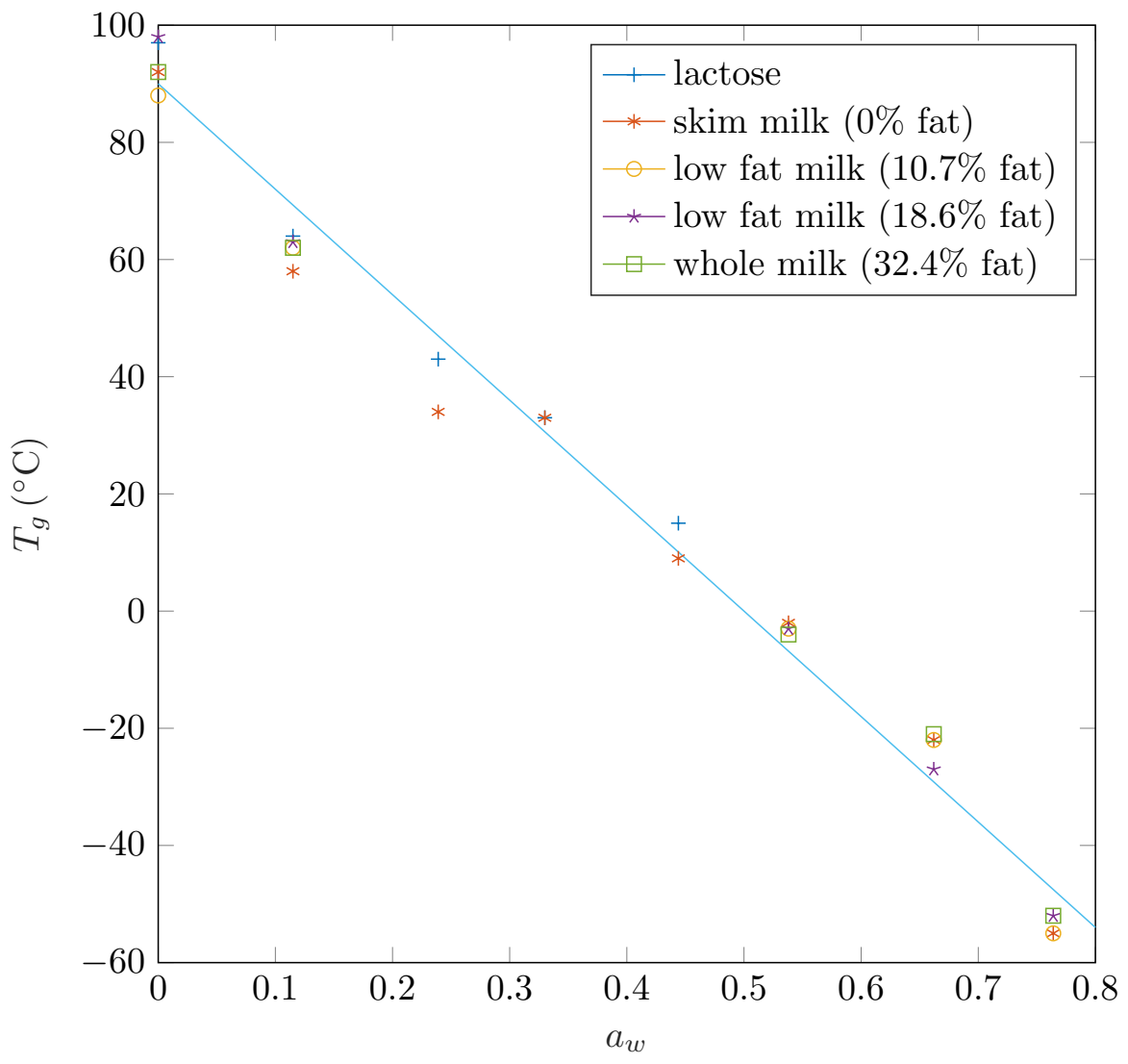


The most extreme conditions might consist of $h_{0}=1$ and $\mathrm{T}=45^{\circ} \mathrm{C}$, whence approximately $h_{c}=0.25$, and this gives an upper limit of

$$
t_{\mathrm{SL}}=\frac{0.11\left(1+\frac{1}{6} \mathrm{k}\right) \mathrm{d}}{h_{\mathrm{b}}} .
$$

With our earlier estimates of $d=0.13 \mathrm{~m}, \mathrm{~h}_{\mathrm{b}}=0.063 \mathrm{~m} /$ year and $\mathrm{k}=\pi$, this leads to $t_{\mathrm{SL}}=4.1$ months. In order to increase this, we recommend increasing the membrane thickness to reduce the seepage rate $h_{b}$. This can also be achieved by increasing the bag dimension, or more generally decreasing the specific surface area, since the vapour pressure in the bag at a fixed time is proportional to this latter quantity.

\subsection{Oxygen transport}

This is similar to moisture transport, but simpler because the concentration of oxygen in the atmosphere does not vary significantly, and the concentration of oxygen in the bags is close to zero. Because of this, the flux of oxygen through the packaging is approximately constant over the life of the milk powder, giving an increase of $5 \%$ by volume of gas in the bags per year for typical packaging.

\section{Permeation model}

We desire to describe the permeation of moisture over time through the packaging membrane of the powder. We track this moisture level and use it as a proxy for shelf life (the time for lactose crystallisation to begin). Many similar models for other products exist in literature and here we modify these for our own purposes. 


\subsection{Assumptions}

The assumptions of our model include the following.

1. The amount (in $\mathrm{kg}$ ) of moisture in the bag increases only due to permeation through the bag membrane.

2. The rate of diffusion of water vapour through the head-space and powder is much larger than the rate through the bag membrane. Therefore we treat the bag as a bulk lot, and ignore transients and spatial variations. The time-scales calculated in Section 2 allow us to use such an assumption.

3. All of the water entering the bag via permeation acts to increase the water activity, $a_{w}$. That is, the vapour pressure of water in the headspace and the powder is assumed to be equal.

4. There is no increase in the water activity due to the release of chemically bound water.

5. The temperature of the powder in the bag is taken to be equal to ambient. We ignore the (rapid [2]) transients in thermal conduction.

6. We model a single bag of powder only, ignoring the effect of stacking, for example, on a pallet of bags, which would necessitate the inclusion of spatial structure.

7. We ignore the effects of chemical reactions which occur slowly, but are important for long time predictions of powder quality.

8. Lactose crystallisation begins once the powder temperature, $T_{p}$, exceeds the glass transition temperature, $T_{g}$, for the current value of water activity, $\mathrm{a}_{w}$, in the bag.

9. Permeation proceeds at steady state and is uniform over the packaging surface area. The bag permeability is independent of the ambient conditions. 
10. The bulk density and structure of the powder is uniform.

\subsection{Model equations}

There exist various models of various complexity for the permeation of water vapour through food packaging $[4,8,9,13,14,16,4,18,19]$. Here we present the governing equations for mass transfer over the packaging interface based mostly on the work by Kruger [13].

Let $p_{\mathrm{av}}$ and $p_{\mathrm{bv}}$ be the vapour pressure $(\mathrm{Pa})$ of water vapour of the atmosphere (outside of the bag) and of the powder inside the bag respectively. In terms of the ambient relative humidity, $h_{0}$, and the water activity in the bag, $a_{w}$,

$$
p_{\mathrm{av}}=p_{\mathrm{sv}} h_{0}, \quad p_{\mathrm{bv}}=p_{\mathrm{sv}} \mathrm{a}_{w}
$$

where $p_{\mathrm{sv}}=p_{\mathrm{sv}}(T)$ is the pressure of water vapour at the saturation condition. The water activity is written as $a_{w}=p_{\text {bv }} / p_{\mathrm{sv}}$.

With the above definitions, we write the governing equation of water vapour transport across the bag membrane as

$$
\frac{d}{d t}\left(m_{w v}\right)=\frac{k}{\delta} A p_{s v}\left(h_{0}-a_{w}\right),
$$

where $t$ is the time in days, $m_{w v}$ is the mass of water vapour in the bag $(\mathrm{kg})$, $\delta$ is the thickness of the bag membrane $(m)$, and $A$ is the surface area of the bag $\left(\mathrm{m}^{2}\right)$. The permeability of the bag membrane, $\mathrm{k}\left(\mathrm{kg} \mathrm{m}^{-1}\right.$ day $\left.{ }^{-1} \mathrm{~Pa}^{-1}\right)$, is in practice temperature dependent (usually written using an Arrhenius-type function, [9]); however, we had only limited experimental data for the Fonterra bags, hence $k$ was considered a constant in this work.

The pressure $(\mathrm{Pa})$ of water vapour at the saturation condition (Bronlund and Paterson [1])

$$
p_{\mathrm{sv}}=\exp \left(23.4795-\frac{3990.56}{\mathrm{~T}+233.833}\right)
$$


where the temperature $\mathrm{T}$ is in ${ }^{\circ} \mathrm{C}$. There are many other relations for the saturation pressure, some of which are outlined in Appendix A.

The water activity in the powder, $\mathrm{a}_{w}$, is related to the powder water content, $\mathrm{X}$ (kg water $/ \mathrm{kg}$ solids), by the GAB sorption isotherm model. We were not able to find any data for milk powders, but did find data for lactose powders [6]. Further experiments would be needed to refine the model for specific dairy powders and specific temperatures. The GAB model relates $a_{w}$ to $X$ via

$$
X=\frac{100 X_{0} \mathrm{Cfa}_{w}}{\left(1-\mathrm{fa}_{w}\right)\left[1+(\mathrm{C}-1) \mathrm{fa}_{w}\right]},
$$

where the constants are given by Foster, Bronlund, and Paterson [6]: $X_{0}=$ $6.27, f=1.01, C=2.81$. With knowledge of the current mass of water vapour in the bag, $m_{\mathrm{wv}}$, we divide this by the mass of the powder, $m_{p}=25 \mathrm{~kg}$, to find $\mathrm{X}$ at any time.

Given appropriate atmospheric and initial conditions, Equations (20)-(22) are sufficient to calculate the water activity, $\boldsymbol{a}_{w}$, of the powder at any given time. We then must approximate the time at which the powder crosses the glass transition temperature threshold.

The data, shown in Figure 1 and from experiments by Jouppila and Roos [11], approximate a function relating the glass transition temperature to the water activity. For simplicity, a linear relationship is chosen;

$$
\mathrm{T}_{g}=88-172.55 \mathrm{a}_{w} .
$$

Initial conditions were specified as the initial temperature, relative humidity and water activity in the powder. These parameters were varied and are discussed in the next section. For the parameter values in Table 4 in the appendix, Equation (20) was solved using ODE45 in Matlab over time for the current temperature and water activity, and the intersection with the glass transition point from Equation (23) calculated to approximate the lactose crystallisation initiation point. Results for constant and varying temperatures are presented in the following Section 3.3. 


\subsection{Results}

We split our results into two sections; the first contains predictions of shelf life at constant temperature and humidity, the second relates to temperature data obtained by Fonterra during the shipment of a powder sample to an African country. Unfortunately there is no humidity data, so we assume constant humidity.

\subsubsection{Constant temperature and humidity}

As an example calculation, we first assume that the ambient humidity and temperature are constants and predict the shelf life (time to lactose crystallisation initiation). This shelf life prediction does not account for changes in the powder due to chemical reactions. Although the rate of these reactions are generally slow, this assumption may produce errors in the prediction for long storage times. Figure 2 displays an example calculation of shelf life with the ambient conditions $\mathrm{T}=20^{\circ} \mathrm{C}$ and $h_{0}=0.8$, which is not unreasonable for summer in New Zealand. The predicted shelf life is just over two years which seems to be of the correct order.

To further understand the effect of temperature, humidity, and the initial water activity, $\mathrm{a}_{w}$, the predicted shelf life was calculated at temperatures between $15^{\circ} \mathrm{C}$ and $52.5^{\circ} \mathrm{C}$, and for humidities between $25 \%$ and $99 \%$. The initial water activity was changed between $\mathrm{a}_{w}=0.175,0.2$, and 0.225 . The predicted shelf life time for each of these cases are displayed in Tables 1 to 3, and in Figure 3.

From Figure 3 the shelf life is roughly exponentially dependent on the storage temperature (and also humidity, not directly shown), with larger temperatures producing drastically lower shelf life times. Even a small change in the storage temperature may affect the shelf life greatly. This effect may be magnified if temperature dependence of permeability was accounted for. Even at $99 \%$ humidity a powder stored at $20^{\circ} \mathrm{C}$ is predicted to last over 600 days without 
Figure 2: Shelf life numerical experiment with constant temperature, $20^{\circ} \mathrm{C}$, and humidity, $80 \%$. The blue curve represents the state of the powder $\left(a_{w}, T\right)$ at time $t$. Once the blue curve intersects the glass transition curve (red) then the time is given as an approximation of shelf life.

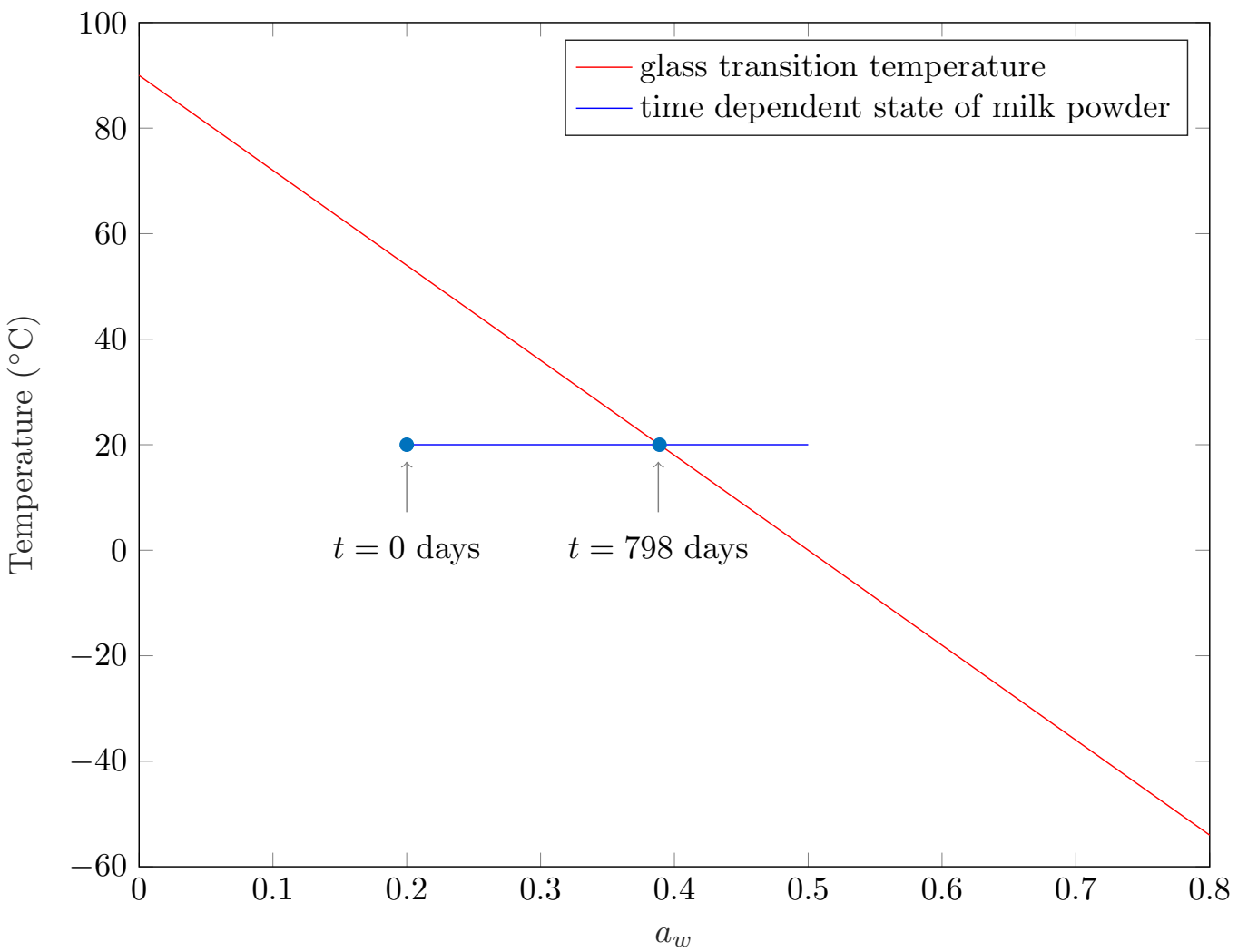


Figure 3: The dependence of shelf life on temperature and relative humidity $(\mathrm{RH})$. The trajectories start with water activity value $\mathrm{a}_{w}=0.2$. The relationship is exponential and shows the importance of maintaining the powder at a low temperature.

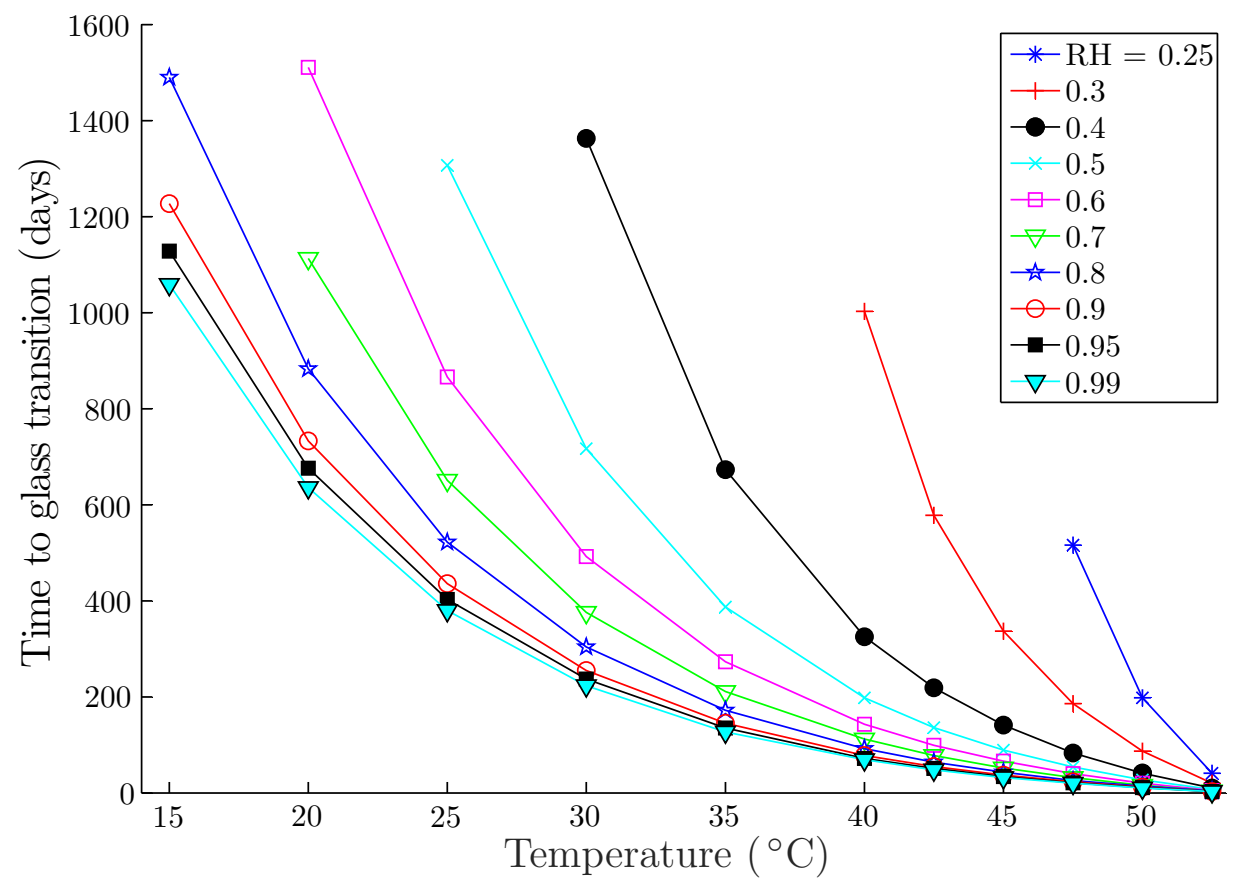

the initiation of crystallisation. The tables are instructive to understand the effect of the initial water activity. For storage temperatures of $30^{\circ} \mathrm{C}$ or less, the difference in storage times between the three initial water activities, $a_{w}=0.175,0.2$, and 0.225, are less than 15\%. Subject to checking that reaction rates are not severely affected, this suggests that money could be saved when the powder is being sent to a temperate country (such as New Zealand), since less moisture needs to be removed during processing. However, for hot and humid countries, reducing the initial water activity below 0.2 generates a significantly longer shelf life, with the relative improvement increasing for 
Table 1: Time (days) until glass transition temperature reached $\left(a_{w}^{0}=0.225\right)$

\begin{tabular}{|l|rrrrrrrrrr|}
\hline & \multicolumn{10}{|c}{$\mathrm{h}_{0}(\%)$} \\
$\mathrm{T}\left({ }^{\circ} \mathrm{C}\right)$ & 25 & 30 & 40 & 50 & 60 & 70 & 80 & 90 & 95 & 99 \\
\hline 15 & - & - & - & - & - & 1760 & 1373 & 1127 & 1034 & 971 \\
20 & - & - & - & - & 1381 & 1010 & 798 & 660 & 607 & 571 \\
25 & - & - & - & 1178 & 770 & 575 & 459 & 382 & 352 & 332 \\
30 & - & - & 1216 & 621 & 421 & 319 & 257 & 215 & 199 & 188 \\
35 & - & - & 561 & 314 & 219 & 168 & 136 & 115 & 106 & 101 \\
40 & - & 819 & 240 & 142 & 101 & 79 & 65 & 55 & 51 & 48 \\
42.5 & - & 418 & 144 & 88 & 63 & 49 & 40 & 34 & 32 & 30 \\
45 & 1734 & 196 & 75 & 47 & 34 & 27 & 22 & 19 & 17 & 17 \\
47.5 & 216 & 61 & 25 & 16 & 12 & 10 & 8 & 7 & 6 & 6 \\
50 & 0 & 0 & 0 & 0 & 0 & 0 & 0 & 0 & 0 & 0 \\
52.5 & 0 & 0 & 0 & 0 & 0 & 0 & 0 & 0 & 0 & 0 \\
\hline
\end{tabular}

larger temperatures (at $50^{\circ} \mathrm{C}$ the shelf life is doubled with $\mathrm{a}_{w}=0.175$ rather than $\mathrm{a}_{w}=0.2$ ). This suggests one way that Fonterra could extend the shelf life in these countries and reduce the amount of rejected product. Indeed, it is necessary to ensure that other problems such as Maillard browning are avoided when reducing this water activity, and the extra cost would have to be compared to the current cost of rejected powders.

\subsubsection{Example measured temperature}

As part of an effort to understand the shelf life in other areas of the world, especially hot and humid areas, Fonterra conducted a temperature experiment on a shipment of powder which was sent to a country in Africa. Unfortunately, humidity was not also measured, but historical meteorological data allowed us to approximate the humidity in this area of the world as $80 \%$ during that 
Table 2: Time (days) until glass transition temperature reached $\left(a_{w}^{0}=0.2\right)$

\begin{tabular}{|l|rrrrrrrrrr|}
\hline & \multicolumn{10}{|c|}{$\mathrm{h}_{\mathrm{o}}(\%)$} \\
$\mathrm{T}\left({ }^{\circ} \mathrm{C}\right)$ & 25 & 30 & 40 & 50 & 60 & 70 & 80 & 90 & 95 & 99 \\
\hline 15 & - & - & - & - & - & - & 1490 & 1227 & 1128 & 1059 \\
20 & - & - & - & - & 1511 & 1113 & 883 & 733 & 676 & 636 \\
25 & - & - & - & 1307 & 866 & 651 & 522 & 436 & 403 & 380 \\
30 & - & - & 1363 & 717 & 492 & 376 & 304 & 255 & 237 & 223 \\
35 & - & - & 673 & 387 & 273 & 211 & 172 & 145 & 135 & 127 \\
40 & - & 1003 & 325 & 198 & 143 & 112 & 92 & 78 & 72 & 69 \\
42.5 & - & 578 & 219 & 136 & 99 & 78 & 64 & 55 & 51 & 48 \\
45 & - & 337 & 141 & 89 & 66 & 52 & 43 & 37 & 34 & 32 \\
47.5 & 516 & 186 & 83 & 54 & 40 & 32 & 26 & 23 & 21 & 20 \\
50 & 198 & 87 & 41 & 27 & 20 & 16 & 14 & 12 & 11 & 10 \\
52.5 & 41 & 20 & 10 & 7 & 5 & 4 & 4 & 3 & 3 & 3 \\
\hline
\end{tabular}

specific month. The temperature data is shown in Figure 4. It is evident that this shipment of powder was exposed to extreme and highly variable temperature conditions. In order to approximate the shelf life of powder in this situation we assume an initial water activity of $\mathrm{a}_{w}=0.2$ and solve Equation (20) using the measured temperature data and a constant $h_{0}=0.8$. Figure 5 shows the $\left(T, a_{w}\right)$ curve for this situation. We observe that the first time that the glass transition temperature curve is intersected is after a month which is due to the extreme temperature that particular day $\left(>55^{\circ} \mathrm{C}\right)$. The curve then dips below the glass transition curve. It is unclear whether moving below the glass transition temperature after exceeding $T_{g}$ will cause lactose crystallisation to cease, or to continue unhindered. The results do show the usefulness our method may be to Fonterra, especially once the humidity data is also available. A bag in the centre of a pallet may not be as susceptible to such extreme temperature variability as it is somewhat insulated by the other 
Table 3: Time (days) until glass transition temperature reached $\left(a_{w}^{0}=0.175\right)$

\begin{tabular}{|l|rrrrrrrrrr|}
\hline & \multicolumn{10}{|c|}{$\mathrm{h}_{\mathrm{o}}(\%)$} \\
$\mathrm{T}\left({ }^{\circ} \mathrm{C}\right)$ & 25 & 30 & 40 & 50 & 60 & 70 & 80 & 90 & 95 & 99 \\
\hline 15 & - & - & - & - & - & - & 1601 & 1322 & 1217 & 1144 \\
20 & - & - & - & - & 1631 & 1210 & 964 & 802 & 741 & 698 \\
25 & - & - & - & 1424 & 955 & 722 & 582 & 487 & 451 & 425 \\
30 & - & - & 1492 & 805 & 558 & 429 & 349 & 294 & 272 & 257 \\
35 & - & - & 770 & 453 & 323 & 251 & 206 & 174 & 162 & 153 \\
40 & - & 1143 & 399 & 248 & 181 & 142 & 117 & 100 & 93 & 88 \\
42.5 & - & 701 & 283 & 180 & 132 & 105 & 87 & 74 & 69 & 65 \\
45 & - & 445 & 198 & 128 & 95 & 75 & 63 & 54 & 50 & 47 \\
47.5 & 689 & 281 & 133 & 88 & 66 & 53 & 44 & 38 & 35 & 33 \\
50 & 351 & 171 & 85 & 57 & 43 & 35 & 29 & 25 & 23 & 22 \\
52.5 & 176 & 95 & 49 & 34 & 26 & 21 & 17 & 15 & 14 & 13 \\
\hline
\end{tabular}

powder. If such large temperatures are common and produce faulty product, then it may be worth investing in some better storage facilities.

\section{Using compaction to reduce milk powder spoilage}

Air is trapped in milk powder which contains moisture and oxygen that degrade the powder over time. This occurs through processes such as lipid oxidation (which is at its greatest when water activity, $a_{w}$ is less than 0.2 or exceeds 0.4), browning and caking, the onset of which occurs at higher $a_{w}$. The package is flushed with Nitrogen after being filled with powder, which removes much of the air; however, about $2 \%$ oxygen remains in the powder. 
Figure 4: Measured temperature data for a powder sample which was sent to a country in Africa. Collected during a pilot experiment by Fonterra.

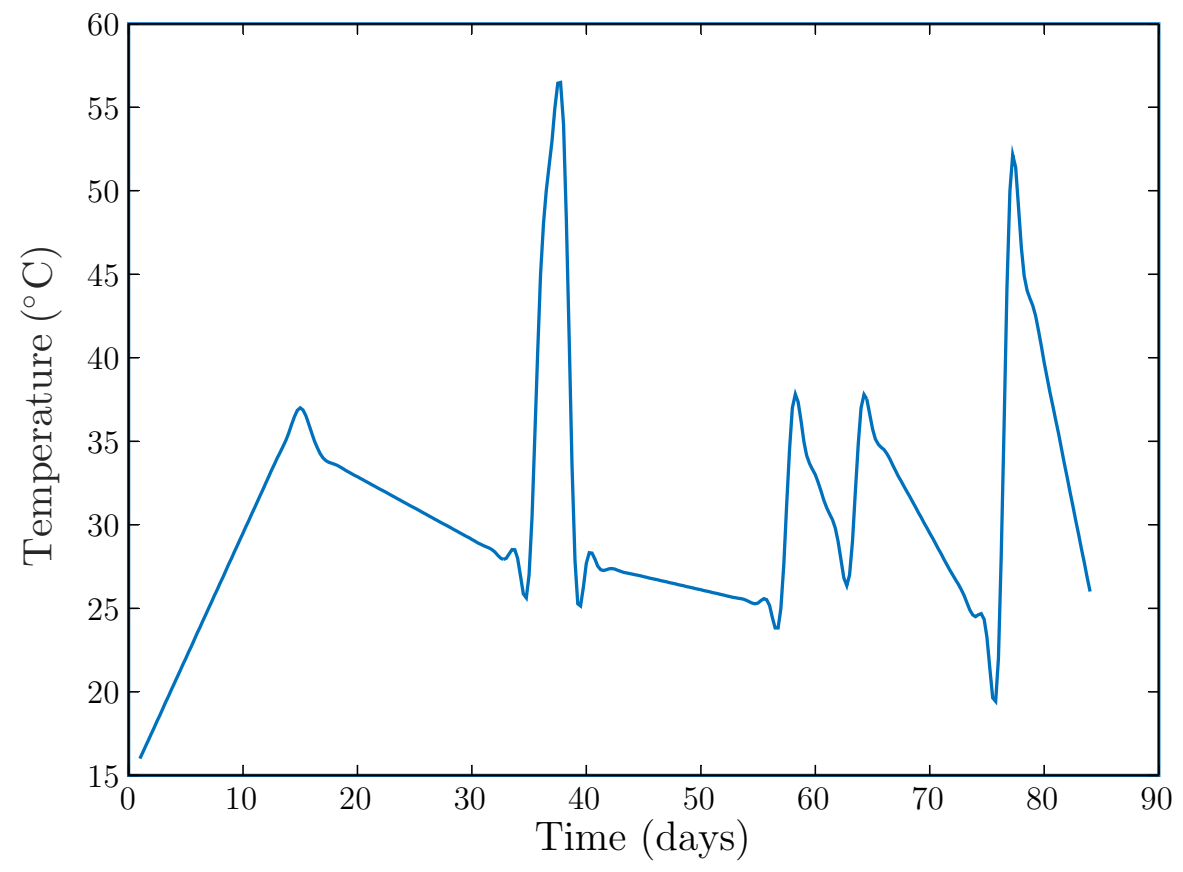

This remains a problem, since very little oxygen is required to produce offflavours through oxidation. One strategy is to prevent additional air from leaking into the packages. Another, perhaps complementary approach, is to find ways of further removing trapped air from the powder. This could be done by further flushing of Nitrogen or Carbon Dioxide through the powder, although this may present difficulties with regards to channelling and the pressure requirements. Another approach would be to reduce the void space within the powder, which is commonly done with soils in geotechnical engineering, as well as for encapsulated medicines in the pharmaceutical industry. This could be done in two ways, either direct compression, or 
Figure 5: Prediction of shelf life for the African temperature data.

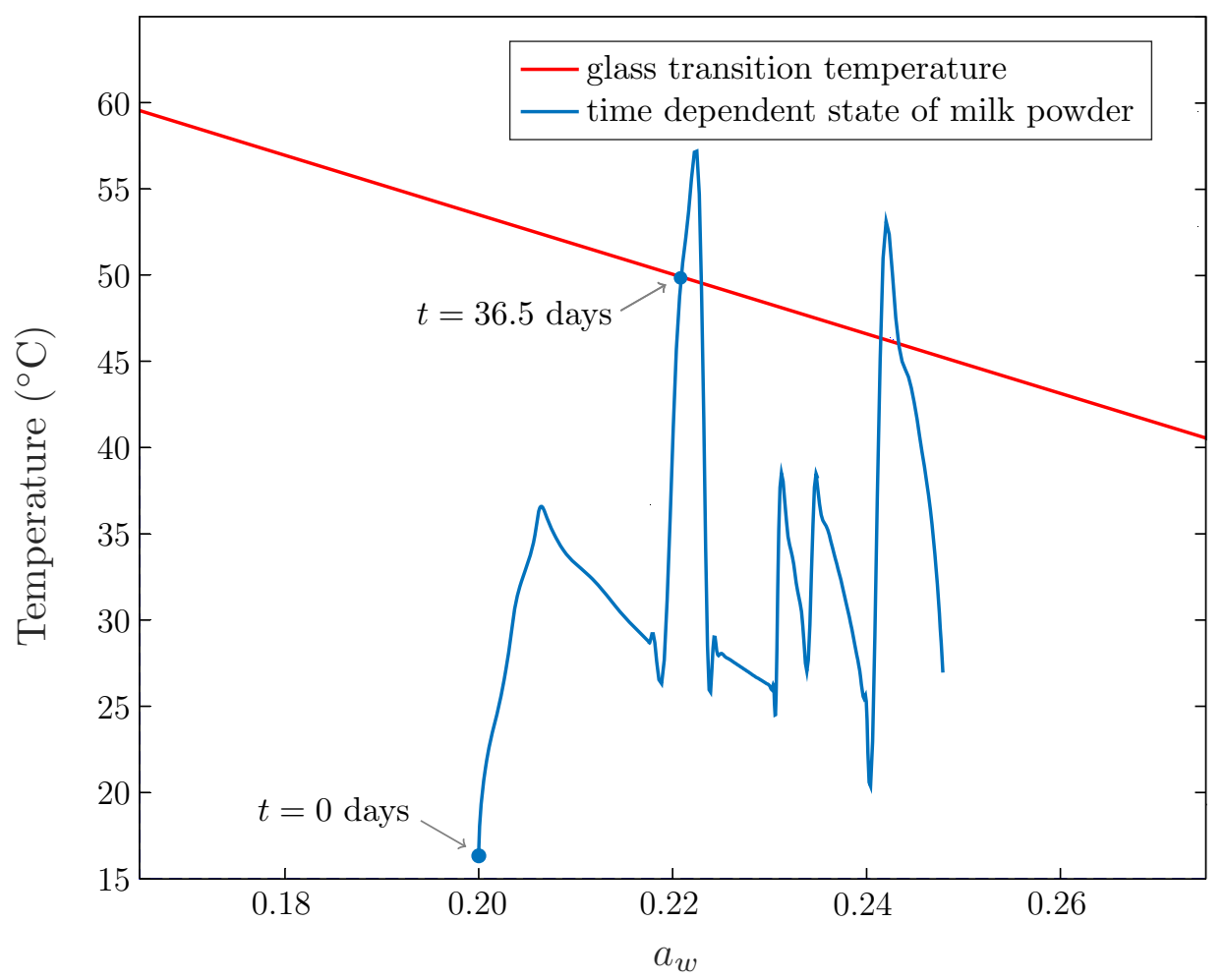

through vibro-compaction. Schematics of these are shown in Figure 6.

\subsection{Compression}

Applied pressure, $\mathrm{P}_{\mathrm{F}}$, leads to change in relative bulk density, $\rho$ (which is directly related to void space within the powder).

There are two classical models which describe the change in bulk density as a function of applied pressure. Firstly, the Kawakita Model [12], which in 
Figure 6: Using (a) compression or (b) vibration to get compaction.

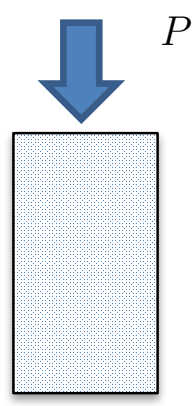

(a)

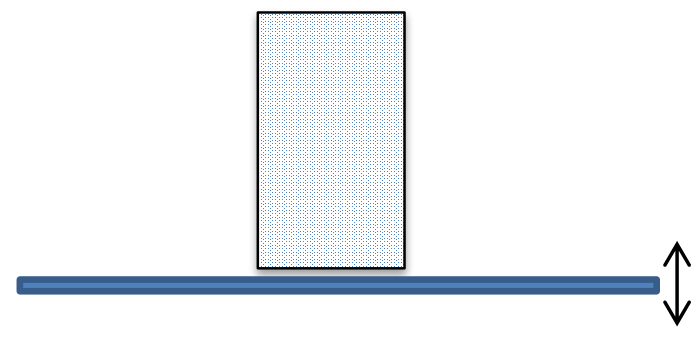

(b)

experiments has been shown to work well at lower applied pressures,

$$
\Delta \mathrm{P}_{\mathrm{F}}=\frac{\Delta \rho-1}{1-\mathrm{A}_{\mathrm{c}} \Delta \rho},
$$

where $\Delta \mathrm{P}_{\mathrm{F}}$ and $\Delta \rho$ are the changes to $\mathrm{P}_{\mathrm{F}}$ and $\rho$, and $A_{\mathrm{c}}$ is an empirically determined constant. Also, the Heckel Model [10], which works better at higher applied pressures,

$$
\frac{d \rho}{d P_{F}}=k_{c}(1-\rho),
$$

where $\boldsymbol{k}_{\boldsymbol{c}}$ is an empirically determined constant. The differential equation (24) has solution

$$
\rho=1-\mathrm{C}_{0} e^{-k_{\mathrm{c}} \mathrm{P}_{\mathrm{F}}} .
$$

An example plot of the solution (25) is shown in Figure 7. The constants $k_{c}$, $A_{c}$ and $C_{0}$ are powder specific.

This relatively simple relationship makes it possible to see how much pressure needs to be applied in order to achieve a given reduction in bulk density (although it does not give the timescales required). There is also some literature describing how this process may be enhanced by passing ultrasonic sound through the powder at the same time as compression. 
Figure 7: The relationship between $\mathrm{P}_{\mathrm{F}}$ and $\rho$ for Heckel's model.

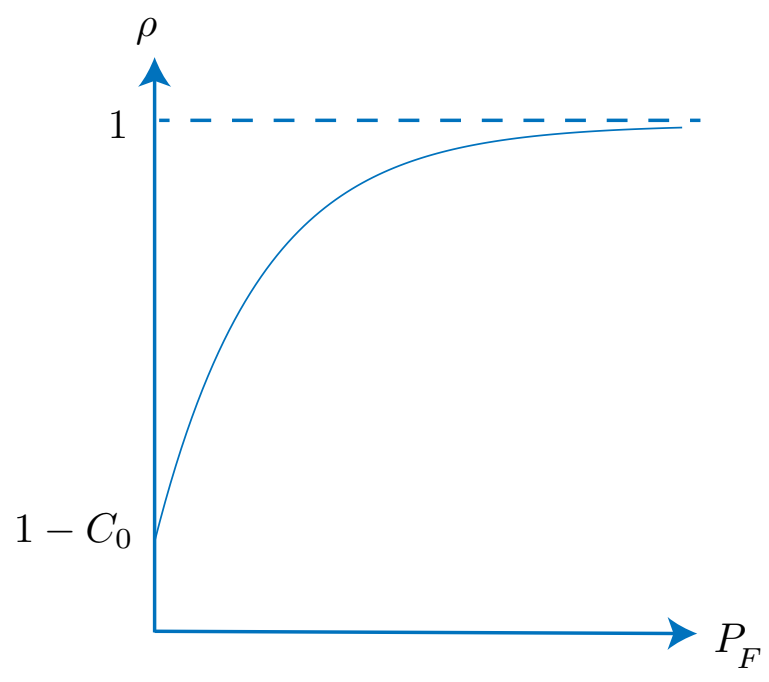

\subsection{Vibro-compaction}

An alternative method for compacting the powder is through vibro-compaction (Figure 6(b)) which oscillates the powder at relatively high frequency. The key parameter is the vibration accelerate,

$$
w=A_{v} \frac{\omega^{2}}{g},
$$

where $A_{v}$ is the amplitude, $\omega=2 \pi f$ is the angular frequency of vibration, $f$ is the frequency of vibration, and $g$ is gravitational acceleration.

Golovanevskiy et al. [7] conducted experiments on vibro-compression using Zirconium-Staurolite mixture of $0.05 \mathrm{~mm}$ to $0.2 \mathrm{~mm}$ particle size range and $2.32 \mathrm{~g} / \mathrm{cm}^{3}$ bulk density. Figure 8 shows their measurements giving relationships between $\rho, f$ and $A_{w}$.

The experiments show that at low values of the frequency $f<1 \mathrm{~Hz}$, the vibration has little effect upon the bulk density. However, in the range 
Figure 8: Relationship between density $\rho$ and vibration frequency $f$ in experiments by Golovanevskiy et al. [7]. Dashed lines indicate key values of $f$.
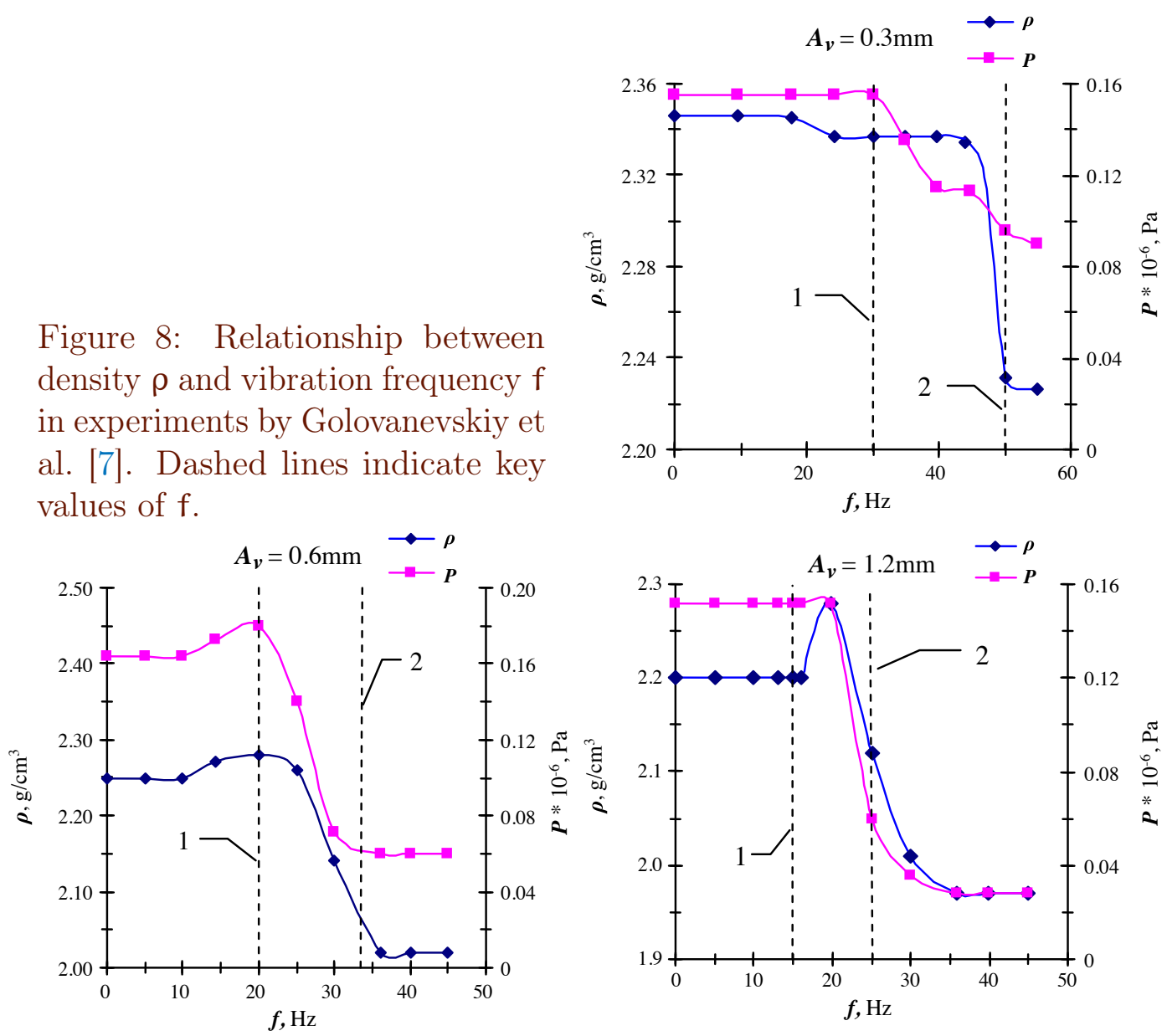
1 to $2 \mathrm{~Hz}$, the vibration leads to either an increase, or decrease, in bulk density, depending upon the value of the amplitude. At the largest amplitude studied in the experiments, this can lead to a $5 \%$ decrease in the void fraction and, presumably, the air content within the granular medium.

\section{$5 \quad$ Statistical approaches}

\subsection{Customer feedback data}

The customer data consists of $\mathrm{N}=232$ records of customer complaints from January 1st 2011 until June 1st 2016. The rather long five year time period was required to acquire the data because the vast majority of product incurs no customer complaints. These complaint records include the following.

- Country of shipment.

- Type of powder:

- Skim Milk, instant and regular;

- Whole Milk, instant and regular;

- Butter Milk.

- Factory which produced the powder.

- Date of manufacture.

- Date of complaint.

\subsubsection{Age}

The difference between the date of manufacture and date of complaint gives an approximate age of the milk powder when it reached the end of its shelf 
Figure 9: Age of powder at time of complaint

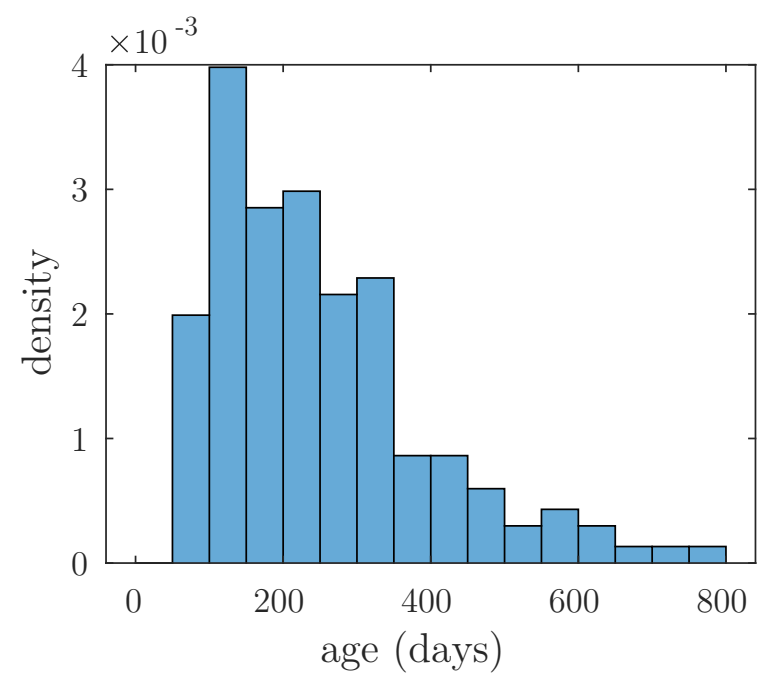

life. As the powder ages there is a progression in the chemical reactions that lead to the milk powder developing unwanted characteristics. We also believe that most powder gets used before it has a chance to age significantly. These two assumptions have the effect of obscuring the relationship between powder reaching the end of its shelf life and age when looking at the absolute number of complaints against age. We lack the information about the total volumes of powder and its age to make inference about the probability of powder being unfit for purpose relative to its age.

The histogram, Figure 9, shows that there are no complaints in the first 50 days after production. The majority of complaints occur with products that are under a year old, between 50 to 350 days. From this it appears as though age itself may not be the cause of the product reaching the end of its shelf life. However, we have no information about the age of powder that is in the market and so no denominator with which to calculate proportions. 


\subsubsection{Country}

The country to which the powder was shipped affects the probability of a complaint occurring. This is a function of both the predominant use of the product in the country as well as the environmental conditions the product may experience in a country. In Australia the product is mostly for commercial use and often is mechanically mixed into large batches and thus the consumer is more accepting of clumping. In African countries the milk is often reconstituted for drinking or home baking and so visual discolouration is more likely to lead to complaints. The differing climatic and industrial conditions in various countries may also be linked to differing rates of complaints. As discussed in Sections 2 and 3, temperature and humidity are linked to powder developing unwanted characteristics, so if the containers were left on the docks in an African country for two months there is a higher likelihood of the powder developing unwanted characteristics than if it were refrigerated in New Zealand.

\subsection{Experimental design}

We designed an experiment to examine which factors cause the $25 \mathrm{~kg}$ bags of milk powder to develop unwanted characteristics.

Cost is an important factor in the design of such an experiment. A single $25 \mathrm{~kg}$ bag of milk powder costs on average $\$ 58$ for whole milk powder, $\$ 47$ for skim milk powder, and $\$ 41$ for butter milk powder (based on the twelve month average of Global Dairy Trade prices from September 2015 to August 2016).

Cost is also affected by the need to maintain ideal experimental conditions. This involves packing the bags on pallets in humidity and temperature controlled containers, the expense of which constrains the maximum number of experimental units we can include.

The number of time measurements taken during the experiment also affects 
cost because measurement involves the destruction of bags.

\subsubsection{Factors}

This experimental design is complicated due to the large number of experimental factors. These include

- Factory (30). There are 30 factories around New Zealand which produce a variety of milk powders. Not every factory produces the same varieties of powder. Each factory receives milk from local suppliers. There are likely to be regional differences in milk. Further variations occur as a result of different equipment that factories use, such as the size and type of dryers.

- Season (3). Early, mid and late season milk.

- Humidity. The humidity in which the packages are stored.

- Temperature. The temperature in which the packages are stored

- Packing (56 bags per pallet). The bags are packed onto a pallet before shipping. The location of a bag on a pallet influences its environmental conditions which affect the time the powder takes to develop unwanted characteristics.

- Age. The time since the powder was manufactured, the longer this is the more likely the powder is to be unfit for purpose.

- Powder type. Skim Milk, instant and regular; Whole Milk, instant and regular; Butter Milk.

\subsubsection{Pilot study}

The decision was made to initially design a pilot study to roughly identify the age at which powders develop unwanted characteristics. This will allow 
us to reduce the number of bags required for the full experiment by focusing on the destructive testing of the bags at an age for which they are likely to have started to develop unwanted characteristics.

We wish to conduct a pilot study to determine the critical points for the powders. This will involve testing a subsection of the powders at a range of temperatures/humidities. Ideally this would consist of using two powders from one factory. The first being a powder which is thought to be resistant to lumping. The second being a powder which is prone to developing unwanted characteristics.

We will place twelve bags of each of these powders into environments with $4 \times 4$ combinations of temperature and humidity. We will test a bag every two months. If for two consecutive months a powder in a location has developed unwanted characteristics, then all remaining bags will be removed from storage and the experiment on that temperature and humidity combination ended.

The experiment would ideally be carried out in a large enough environment to control the temperature and humidity of the environment. We envision using a refrigerated container which has been modified to allow for humidity control. The bags of milk powder will be stacked on pallets in the same way milk powders are packed commercially. We will randomise the order of the bags to control for the effects of packing location.

\subsubsection{Logistics}

We are working on the assumption that we can convert ten foot refrigerated containers to maintain a consistent heat and humidity. Each container will maintain a constant temperature and humidity. We are intending to have:

- four unique temperature levels;

- four unique humidity levels.

We are intending to use a partial factorial design with these levels requiring 
eight containers. There will be two replications of each temperature and each humidity in a $4^{4-2}$ factorial design. We estimate that the container will be able to hold ten pallets, each pallet holds 56 bags of milk powder and thus each container holds 560 bags of powder. Thus, the cost of powder for the pilot study will be around $\$ 250,000$.

\subsubsection{Time}

Physical and sensory variables of the powder will be measured every three months for the first year, as we do not expect the powder to develop unwanted characteristics prior to this. In the second year the intervals between time points will be reduced to every two months until the trial ends at 24 months as this is when the key changes in the powder are most likely to occur. These intervals can be varied to allow closer time points for the samples exposed to higher temperature and humidity conditions. This will give us 56 bags at each time point. There is potential to include in this a limited sample of other powder type as well.

An option for generating a baseline control condition against which to compare the experimental data is to use retained powder from around the country. Retained powder occurs when a bag is not shipped for various reasons, including over supply.

The retained powder will have been stored in relatively consistent temperatures and humidities at the various factories. Examining the powder at its current stage will allow an observational analysis of the age at which powders are unfit for market. The advantages of using the retained powder are that there are no storage costs and the retained powder has already been written off, and may give us an indication of the average shelf life of the product. The disadvantage of this approach comes from the lack of experimental control. 


\section{Discussion and conclusions}

The study and prediction of the shelf-life of milk powders is a complicated endeavour. The challenge presented at the 2016 MINZ study group to predict this shelf life was attended by various people of different backgrounds. As such, the problem has been approached in a number of ways.

Section 2 showed the thermal transport through a bag of powder to be significantly shorter than the storage time scale. This justifies the assumption that the temperature of a bag was uniform, which greatly simplified the analysis. A model of moisture diffusion through the product was developed and an analytical solution found. The predicted shelf life using this method was in good agreement with those of Section 3

Section 3 developed a simple mass transfer model of water vapour through the bag membrane which predicts the water activity as a function of time in a bag of powder. The definition of shelf life used in this section was the time for the powder temperature $T$ to intersect the glass transition temperature curve $T_{g}$ (which is a function of the water activity $a_{w}$ ). The full list of chemical reactions are complex and were not accounted for. Our predictions may become less reliable over longer storage times when chemical reactions are expected to become more important. We have shown the dependence of this measure of shelf life on the initial water activity, $\mathbf{a}_{w}$, the storage temperature, $T$, and the ambient relative humidity, $h_{0}$. The temperature was observed to have a large effect on the shelf life. For temperate climates it may be possible to allow the water activity to start at a larger value in order to reduce drying costs. However, there is a significant extension in shelf life with a lower water activity when the temperature is large $\left(\approx 40^{\circ} \mathrm{C}\right)$. This would reduce the failure rate of the powder, but would be more expensive to produce. These results need to be contextualised, since we did not account for chemical reactions in the medium which are affected by changing the initial water content.

Further methods to extend shelf life in hot and humid countries include 
increasing the thickness of the bag membrane (Equation (20) predicts a doubling of the shelf life with doubling the thickness of the bag), decreasing the permeability of the bag membrane by using some other material, insulating the transport container, or increasing the volume to surface area ratio of the powder. Such suggestions need to be examined by Fonterra for financial benefit.

Section 4 suggests that compaction, either by directly compressing a bag of powder or by a vibration process, may reduce the residual oxygen in the product. Such a reduction in residual oxygen is hypothesised to reduce the rate of lipid oxidation and extend the shelf life of the powder. A literature study was presented and estimated that an approximate 5\% decrease in residual oxygen was theoretically possible. The extent to which this reduction will help to increase shelf life is unclear and further investigation is needed.

A statistical analysis of complaints data was performed in Section 5, and a pilot study was designed. For the complaints data the season of milk, age of the powder, and destination country of sale were considered. There appeared to be an excess of complaints from late season milk, but not enough volume of production data was available to draw strong conclusions. Most of the complaints data arose less that one year after manufacture, but again data was lacking about proportions of ages of milk in the marketplace. Many complaints were from high temperature and high humidity equatorial countries. This suggests that powder storage may be the main influence on shelf life of powders in these regions. The pilot study was of fractional factorial split plot type and accounted for temperature, humidity, and storage time. This was a balance between accounting for the expected important factors and the cost of such a study. This is an ongoing endeavour at Fonterra.

Finally, we outline some recommendations to Fonterra to help better understand this aspect of shelf life and help reduce wasted product.

1. Conduct tests on the bag membrane to determine the temperature dependence of water vapour permeability. In this work a single permeability value was used. It is expected that more data will improve the predic- 
tions of this model, particularly at extreme temperatures.

2. Conduct tests on the bag membrane to determine the temperature dependence of oxygen permeability. Since oxidation is a major cause of powder failure, this problem should be analysed, and can be modelled in a similar way to the permeation of water vapour.

3. Experimentally determine sorption isotherms for the various milk powders. In the current work the isotherm for lactose was used (Equation (22)) which contains a certain amount of error compared to specific dairy powders.

4. Examine the cost and chemical effect of lowering the initial water activity for very hot climates, and increasing it for temperate climates. There is some potential to improve the shelf life in extreme temperatures, but further work needs to be undertaken.

5. Examine the cost of using a new bagging material with lower permeability, or using a thicker bag membrane.

6. Further develop models which include temporal and spatial variations in temperature and moisture. Such a model is likely to be necessary when examining a pallet of bags of powder, rather than a single bag as in this study.

7. Collect a list of the main chemical reactions which control quality in the various milk powders. Furthermore, examine the rate of reaction as a function of temperature. Such information will allow a more detailed model of the aging of milk powder which will lead to more accurate predictions of shelf life.

8. Attach temperature and humidity sensors to powder samples shipped to hot and humid countries. Since the permeation of moisture is dependent on both temperature and humidity, such sensors are necessary to obtain accurate shelf life predictions. 


\section{A Parameters}

Table 4: Parameters for Sections 2 and 3

\begin{tabular}{|c|c|c|c|}
\hline Symbol & Quantity & Unit & Value \\
\hline$p_{\mathrm{av}}$ & $\begin{array}{l}\text { Vapour pressure of water in } \\
\text { the atmosphere. }\end{array}$ & $\mathrm{Pa}$ & \\
\hline$p_{b v}$ & $\begin{array}{l}\text { Vapour pressure of water in } \\
\text { the bag. }\end{array}$ & $\mathrm{Pa}$ & \\
\hline$p_{\mathrm{sv}}$ & $\begin{array}{l}\text { Saturation vapour pressure } \\
\text { of water. }\end{array}$ & $\mathrm{Pa}$ & Eq. (21) \\
\hline$p$ & Vapour partial pressure & $\mathrm{Pa}$ & \\
\hline$p_{p}$ & Pellet vapour pressure. & $\mathrm{Pa}$ & \\
\hline$p_{c}$ & $\begin{array}{l}\text { Critical vapour pressure in } \\
\text { bag at glass transition. }\end{array}$ & $\mathrm{Pa}$ & \\
\hline$h_{0}$ & $\begin{array}{l}\text { Relative humidity of atmo- } \\
\text { sphere. }\end{array}$ & & \\
\hline$h_{c}$ & Critical relative humidity. & & \\
\hline$a_{w}$ & $\begin{array}{l}\text { Water activity in the pow- } \\
\text { der. }\end{array}$ & & Eq. (22) \\
\hline $\mathrm{m}_{\mathrm{wv}}$ & $\begin{array}{l}\text { Mass of water vapour in the } \\
\text { bag at time } t \text {. }\end{array}$ & $\mathrm{kg}$ & Eq. (20) \\
\hline $\begin{array}{c}m_{p} \\
t\end{array}$ & $\begin{array}{l}\text { Mass of powder in the bag. } \\
\text { Time. }\end{array}$ & $\begin{array}{l}\mathrm{kg} \\
\text { years in Section 2, } \\
\text { days otherwise. }\end{array}$ & 25 \\
\hline$t_{T}$ & Thermal time scale. & hours & \\
\hline$t_{b}$ & Bag diffusion time scale. & $\mathrm{s}$ & \\
\hline$t_{p}$ & Pellet diffusion time scale. & & \\
\hline $\begin{array}{c}\mathrm{t}_{\mathrm{SL}} \\
\mathrm{d}\end{array}$ & $\begin{array}{l}\text { Product shelf life. } \\
\text { Bag thickness. }\end{array}$ & $\begin{array}{l}\text { years } \\
\mathrm{m}\end{array}$ & 0.13 \\
\hline$d_{p}$ & Pellet diameter. & $\mathrm{m}$ & \\
\hline$r$ & Pellet radius. & $\mathrm{m}$ & \\
\hline
\end{tabular}




\begin{tabular}{|c|c|c|c|}
\hline$s$ & Specific surface area of pel- & $\mathrm{m}^{-1}$ & \\
\hline$k_{s}$ & $\begin{array}{l}\text { lets. } \\
\text { Specific surface area con- } \\
\text { stant. }\end{array}$ & & $\pi$ \\
\hline $\mathrm{D}$ & $\begin{array}{l}\text { Diffusion coefficient on bag } \\
\text { boundary. }\end{array}$ & $\mathrm{m}^{2} \mathrm{~s}^{-1}$ & $10^{-5}$ \\
\hline $\mathrm{D}_{\mathrm{p}}$ & $\begin{array}{l}\text { Diffusion coefficient in pel- } \\
\text { lets. }\end{array}$ & $\mathrm{m}^{2} \mathrm{~s}^{-1}$ & \\
\hline$h_{b}$ & Mass transfer coefficient. & $\mathrm{m} /$ year & 0.063 \\
\hline$q$ & Vapour flux to pellets & $\operatorname{kg~s}^{-3}$ & \\
\hline K & Thermal diffusivity. & $\mathrm{m}^{2} \mathrm{~s}^{-1}$ & $10^{-5}$ \\
\hline k & $\begin{array}{l}\text { Permeability of bag mem- } \\
\text { brane. }\end{array}$ & $\mathrm{kg} \mathrm{m}^{-1} \mathrm{day}^{-1} \mathrm{~Pa}^{-1}$ & $5.14 \cdot 10^{-11}$ \\
\hline$\delta$ & $\begin{array}{l}\text { Thickness of the bag mem- } \\
\text { brane. }\end{array}$ & $\mathrm{m}$ & $75 \cdot 10^{-6}$ \\
\hline$A$ & Surface area of bag. & $\mathrm{m}^{2}$ & 1.26 \\
\hline $\mathrm{T}$ & Temperature of bag & ${ }^{\circ} \mathrm{C}$ & \\
\hline $\mathrm{T}_{\mathrm{g}}$ & $\begin{array}{l}\text { Temperature of glass transi- } \\
\text { tion. }\end{array}$ & ${ }^{\circ} \mathrm{C}$ & Eq. (23) \\
\hline$X$ & Water content of powder. & $\mathrm{kg}$ water $/ \mathrm{kg}$ solids & $m_{\mathrm{wv}} / \mathrm{m}_{\mathrm{p}}$ \\
\hline$X_{0}$ & GAB constant 1 & $\mathrm{~kg}$ water $/ \mathrm{kg}$ solids & 6.27 \\
\hline C & GAB constant 2 . & & 2.81 \\
\hline$f$ & GAB constant 3 . & & 1.01 \\
\hline$\lambda$ & Constant. & & $1 / 90$ \\
\hline$a_{0}$ & Constant. & & 0.5 \\
\hline
\end{tabular}

\section{References}

[1] John E. Bronlund and A.H.J. (Tony) Paterson. "A model to predict moisture migration in bulk powders subjected to temperature gradients". In: Proceedings of the 11th International Congress on Engineering and Food, Athens, Greece. May 2011. URL: 
Table 5: Table of symbols and constants for Section 4.

\begin{tabular}{|c|l|l|c|}
\hline Symbol & Quantity & Unit & Value \\
\hline$a_{w}$ & Water activity in the powder. & & Eq. (22) \\
$\mathrm{P}_{\mathrm{F}}$ & Applied pressure. & $\mathrm{Pa}$ & \\
$\rho$ & Powder bulk density. & $\mathrm{kg} \mathrm{m}^{-3}$ & \\
$w$ & Vibration accelerate. & $\mathrm{s}^{-1}$ & \\
$\omega$ & Angular frequency of vibration. & $\mathrm{Hz}$ & \\
$f$ & Frequency of vibration. & $\mathrm{m}^{-2}$ & \\
$\mathrm{~A}_{v}$ & Amplitude of vibration. & $\mathrm{ms}^{-2}$ & \\
$g$ & Gravity. & & \\
$A_{c}$ & Constant. & & \\
$k_{c}$ & Constant. & & \\
$C_{0}$ & Constant. & & \\
\hline
\end{tabular}

WWW. icef11.org/content/papers/mcf/MCF624.pdf (cit. on pp. M384, M393).

[2] John E. Bronlund and A.H.J. (Tony) Paterson. "Mathematical modelling of temperature induced moisture migration in bulk powders". In: Chemical Engineering Science 63.9 (2008), pp. 2330-2340. ISSN: 0009-2509. DOI: 10.1016/j.ces.2007.12.021 (cit. on pp. M384, M392).

[3] John E. Bronlund and A.H.J. (Tony) Paterson. "Moisture sorption isotherms for crystalline, amorphous and predominantly crystalline lactose powders". In: International Dairy Journal 14.3 (2004), pp. 247-254. ISSN: 0958-6946. DOI: 10.1016/S0958-6946 (03)00176-6 (cit. on p. M384).

[4] Zhenhui Chu, Lixin Lu, and Jun Wang. "Mathematical Model for Water Transfer in Multidomain Food Packed in Permeable Packaging". In: Packaging Technology and Science 26 (2013), pp. 11-22. ISSN: 1099-1522. DOI: 10.1002/pts. 1992 (cit. on p. M393). 
[5] Kylie D. Foster, John E. Bronlund, and A.H.J. (Tony) Paterson. "Glass transition related cohesion of amorphous sugar powders". In: Journal of Food Engineering 77.4 (2006), pp. 997-1006. ISSN: 0260-8774. DOI: 10.1016/j.jfoodeng.2005.08.028 (cit. on p. M384).

[6] Kylie D. Foster, John E. Bronlund, and A.H.J. (Tony) Paterson. "The prediction of moisture sorption isotherms for dairy powders". In: International Dairy Journal 15.4 (2005), pp. 411-418. ISSN: 0958-6946. DOI: 10.1016/j.idairyj.2004.08.003 (cit. on pp. M384, M394).

[7] Vladimir A. Golovanevskiy et al. "Vibration-induced phenomena in bulk granular materials". In: International Journal of Mineral Processing 100.3-4 (2011), pp. 79-85. ISSN: 0301-7516. DOI: 10.1016/j.minpro.2011.05.001 (cit. on pp. M404, M405).

[8] Tushar Gulati et al. "Modeling moisture migration in a multi-domain food system: Application to storage of a sandwich system". In: Food Research International 76, Part 3 (2015), pp. 427-438. ISSN: 0963-9969. DOI: $10.1016 /$ j.foodres.2015.06.022 (cit. on p. M393).

[9] Fayi Hao, Lixin Lu, and Jun Wang. "Finite Element Simulation of Shelf Life Prediction of Moisture-Sensitive Crackers in Permeable Packaging under Different Storage Conditions". In: Journal of Food Processing and Preservation 40.1 (2016), pp. 37-47. ISSN: 1745-4549. DOI: $10.1111 /$ jfpp.12581 (cit. on pp. M388, M393).

[10] R. W. Heckel. "Density-pressure relationship in powder compaction". In: Trans Met Soc AIME 22 (1961), pp. 671-675 (cit. on p. M403).

[11] K. Jouppila and Y.H. Roos. "Glass Transitions and Crystallization in Milk Powders". In: Journal of Dairy Science 77.10 (1994), pp. 2907-2915. ISSN: 0022-0302. DOI:

10.3168/jds.S0022-0302(94)77231-3 (cit. on pp. M389, M390, M394). 
[12] Kimio Kawakita and Yuhbun Tsutsumi. "A Comparison of Equations for Powder Compression". In: Bulletin of the Chemical Society of Japan 39.7 (1966), pp. 1364-1368. DOI: 10.1246/bcsj.39.1364 (cit. on p. M402).

[13] Jacus Kruger. "Mathematical modelling of mass transfer in food packaging systems". MA thesis. Massey University, Palmerston North, New Zealand, 2011. URL: http://hdl . handle.net/10179/4041 (cit. on pp. M384, M393).

[14] T.P Labuza and C.R Hyman. "Moisture migration and control in multi-domain foods". In: Trends in Food Science 83 Technology 9.2 (1998), pp. 47-55. ISSN: 0924-2244. DOI: 10.1016/S0924-2244(98)00005-3 (cit. on p. M393).

[15] Theodore P. Labuza and L. R. Dugan Jr. "Kinetics of lipid oxidation in foods". In: C R C Critical Reviews in Food Technology 2.3 (1971), pp. 355-405. DOI: 10.1080/10408397109527127 (cit. on pp. M382, M384).

[16] I.S.M. Macedo et al. "Quality by design for packaging of granola breakfast product". In: Food Control 29.2 (2013). Predictive Modelling of Food Quality and Safety, pp. 438-443. ISSN: 0956-7135. DOI: 10.1016/j.foodcont.2012.05.045 (cit. on p. M393).

[17] D.B. Min et al. "Effects of Packaging Conditions on the Flavor Stability of Dry Whole Milk". In: Journal of Food Science 54.5 (1989), pp. 1222-1224. ISSN: 1750-3841. DOI: 10.1111/j.1365-2621.1989.tb05959.x (cit. on p. M382).

[18] Jens Risbo. "The dynamics of moisture migration in packaged multi-component food systems I: shelf life predictions for a cereal-raisin system". In: Journal of Food Engineering 58.3 (2003), pp. 239-246. ISSN: 0260-8774. DOI: 10.1016/S0260-8774(02)00373-4 (cit. on p. M393). 
[19] Elisabeth Roca et al. "Predicting moisture transfer and shelf-life of multidomain food products". In: Journal of Food Engineering 86.1 (2008), pp. 74-83. ISSN: 0260-8774. DOI: 10.1016/j.jfoodeng.2007.09.012 (cit. on p. M393).

[20] Yrjö H. Roos. "Glass Transition Temperature and Its Relevance in Food Processing". In: Annual Review of Food Science and Technology 1.1 (2010). PMID: 22129345, pp. 469-496. DOI: 10.1146/annurev. food.102308.124139 (cit. on p. M385).

[21] Tomoko Shimamura and Hiroyuki Ukeda. "Maillard Reaction in Milk Effect of Heat Treatment". In: Milk Protein. Ed. by Walter Hurley. InTech, 2012, pp. 147-58. DOI: 10.5772/50079 (cit. on p. M382).

[22] ElmiraArab Tehrany and Kees Sonneveld. "Packaging and the Shelf Life of Milk Powders". In: Food Packaging and Shelf Life, A Practical Guide. Ed. by Gordon L. Robertson. CRC Press, 2009, pp. 127-141. ISBN: 978-1-4200-7844-2. DOI: 10.1201/9781420078459-c7 (cit. on pp. M381, M382).

[23] Marie E C Thomas et al. "Milk powders ageing: effect on physical and functional properties". In: Critical Reviews in Food Science and Nutrition 44.5 (2004), pp. 297-322. DOI: 10.1080/10408690490464041 (cit. on pp. M381, M389).

[24] Marianne K Thomsen et al. "Temperature effect on lactose crystallization, maillard reactions, and lipid oxidation in whole milk powder". In: J Agric Food Chem 53.18 (Sept. 2005), pp. 7082-7090. DOI: 10.1021/jf050862p (cit. on pp. M381, M382, M383).

\section{Author addresses}

1. V. Chopovda, Institute of Fundamental Sciences, Massey University, Albany, New ZEaland.

mailto:v.chopovda@massey.ac.nz 
2. R. J. Clarke, Department of Engineering Science, University of Auckland, Auckland, New Zealand. mailto:rj.clarke@auckland.ac.nz orcid:0000-0001-8036-4733

3. A. C. Fowler, University of Oxford, Oxford, UK, \& University of Limerick, IRELAND. mailto : fowler@maths . ox . ac . uk

4. L. A. Fullard, Institute of Fundamental Sciences, Massey University, Palmerston North, New Zealand.

mailto:L.Fullard@massey.ac.nz orcid:0000-0002-9193-8664

5. J. Goodman, Department of Statistics, University of Auckland, Auckland, New Zealand. mailto:jgoo090@aucklanduni.ac.nz

6. L. M. Thomasen, Fonterra Research Centre, Palmerston North, NeW ZeAland.

mailto:Lisa. Thomasen@f onterra.com

7. S. W. Taylor, Department of Mathematics, University of Auckland, Auckland, NEW ZEALAND.

mailto:s.taylor@auckland.ac.nz orcid:0000-0003-1633-3582 